\title{
Animal models for nickel allergy
}

To the Editor - Vemula et al. report that calcium carbonate and calcium phosphate nanoparticles that are about $70 \mathrm{~nm}$ in diameter can capture nickel ions $\left(\mathrm{Ni}^{2+}\right)$ from the surface of the skin ${ }^{1}$. They suggest that applying the nanoparticles to the skin may limit the exposure to - and thereby prevent allergy towards $-\mathrm{Ni}^{2+}$, which can cause skin irritation and inflammation or contact dermatitis. Although many individuals who are sensitive to $\mathrm{Ni}^{2+}$ will benefit from this innovative approach, the animal model used to study the clinical effectiveness of the nanoparticles is problematic.

We have recently shown that the development of nickel allergy requires both an antigen-specific signal that activates $\mathrm{T}$ lymphocytes, and a non-specific pro-inflammatory signal that triggers innate immunity ${ }^{2}$. $\mathrm{Ni}^{2+}$ induces allergy by interacting with the histidine residues at positions 456 and 458 on the innate immune receptor, Toll-like receptor 4 (TLR4). Because these histidines are present in primates but not in mice, only animals that express the human homologue of TLR4 will develop contact dermatitis, whereas animals that express mouse TLR4 will not ${ }^{2}$. However, it is possible to trigger $\mathrm{Ni}^{2+}$ allergy in animals that express mouse TLR4 by coincident application of lipopolysaccharide (LPS) - a classic agonist of TLR4 (ref. 3).

Vemula et al. intended to study nickel allergy in $\mathrm{C} 3 \mathrm{H} / \mathrm{HeJ}$ mice $^{1}$, but this strain contains a mutation at position 712 of the TLR4 that causes the mice to be nonresponsive to LPS. This means that because the LPS-TLR4 signalling in the mice is defective, coincident application of LPS will not trigger $\mathrm{Ni}^{2+}$ allergy ${ }^{4}$. Furthermore, the experimental design did not include an essential control group - mice that were treated with $\mathrm{Ni}^{2+}$ but had not undergone the sensitization procedure. Such a control would allow the discrimination of a genuine hypersensitivity response, which requires the generation of haptenspecific T cells during the sensitization phase, from an irritant toxic effect of $\mathrm{Ni}^{2+}$, which does not rely on the generation of $\mathrm{Ni}^{2+}$-specific $\mathrm{T}$ cells. Therefore, it seems that Vemula et al. have investigated the effect of nanoparticles on $\mathrm{Ni}^{2+}$ toxicity, which occurs by events that are independent of TLR4 (ref. 5), rather than investigating $\mathrm{Ni}^{2+}$ allergy.

In conclusion, adequate allergy models should be used to clarify whether nanoparticles indeed qualify as the desired preventive tool against contact allergy to $\mathrm{Ni}^{2+}$.

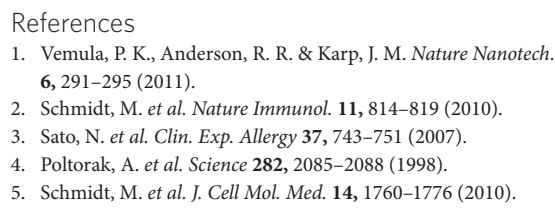

Marc Schmidt', Stefan F. Martin², Marina A. Freudenberg ${ }^{3}$ and Matthias Goebeler ${ }^{1 \star}$ 'Department of Dermatology, University of Giessen, Giessen, Germany, ${ }^{2}$ Allergy Research Group, Department of Dermatology, University Medical Center Freiburg, Freiburg, Germany, ${ }^{3}$ Max-Planck-Institute for Immunobiology and Epigenetics, Freiburg, Germany.

*e-mail: matthias.goebeler@derma.med.unigiessen. de

\section{Authors' reply - The $\mathrm{C} 3 \mathrm{H} / \mathrm{HeJ}$}

mouse model ${ }^{1}$ we used in our paper 'Nanoparticles reduce nickel allergy by capturing metal ions' ${ }^{2}$ is indeed one of many and is not a robust allergy model. Given that nickel-sensitized patients often endure a less heightened response when treated with lower doses of $\mathrm{Ni}^{2+}$ (refs 3,4), the nickel-sensitized mouse model was used to demonstrate that nanoparticles could indeed reduce $\mathrm{Ni}^{2+}$ exposure. As suggested in the correspondence by Schmidt et al., regardless of the host and cutaneous-response pathways, nanoparticles that sequester nickel on the skin surface may offer protection against all such pathways. Our unpublished control experiments on mice ears showed that $\mathrm{Ni}^{2+}$ did not induce inflammation in non-sensitized healthy mice, which is in agreement with non-sensitized humans that typically do not experience a response to nickel ions ${ }^{5}$. It is also important to consider that we observed reactions to nickel only after, not during, nickel sensitization. In addition to showing that nanoparticles significantly reduced the inflammatory response induced by $\mathrm{Ni}^{2+}$ in vivo, our in vitro experiments using inductively coupled plasma atomic emission spectrometry confirmed that the nanoparticles did efficiently capture $\mathrm{Ni}^{2+}$ in solution. Furthermore, energy-dispersive $\mathrm{X}$-ray diffraction analysis of intact skin containing artificial sweat showed that the nanoparticles were able to prevent nickel from going through the skin. By performing inductively coupled plasma atomic emission on dissolved skin that had been treated with nickel, we verified that the nanoparticles can reduce the concentration of nickel in the skin from 400 to $2.5 \mathrm{ppm}$.

In retrospect, a more appropriate title for the paper may have been 'Nanoparticles reduce nickel irritation by capturing metal ions.

References

1. Siller, G. M. \& Seymour, G. J. Australas. J. Dermatol. 35, 77-81(1994).
2. Vemula, P. K., Anderson, R. R. \& Karp, J. M. Nature Nanotech. 6, 291-295 (2011)

3. Frosch, P. J. \& Kligman, A. M. Contact Dermatitis 2, 314-324 (1976).

4. Vandenberg, J. J. \& Epstein, W. L. J. Invest. Dermatol. 41, 419-428 (1963).

5. Seidenari, S., Motolese, A. \& Belletti, B. Contact Dermatitis 34, 88-92 (1996).

Praveen Kumar Vemula, ${ }^{1,2,3}$, R. Rox Anderson ${ }^{4}$ and Jeffrey M. Karp ${ }^{1,2,3 *}$

${ }^{1}$ Center for Regenerative Therapeutics and Department of Medicine, Brigham and Women's Hospital, Harvard Medical School, Boston, Massachusetts 02115 , USA, ${ }^{2}$ Harvard Stem Cell Institute, 1350 Massachusetts Avenue, Cambridge, Massachusetts 02138, USA, ${ }^{3}$ HarvardMIT Division of Health Sciences and Technology, 65 Lansdowne Street, Cambridge, Massachusetts 02139, USA, ${ }^{4}$ Laser and Cosmetic Dermatology Center and Wellman Center for Photomedicine, Massachusetts General Hospital, Harvard Medical School, Boston, Massachusetts 02114, USA.

*e-mail: jkarp@rics.bwh.harvard.edu 